\title{
An easy handling system for installing heavy glass using human robot cooperation
}

\author{
Myeong Su Gil ${ }^{1}$, Sang Ho Kim², Hyeon Guk Kim ${ }^{1}$, Min Sung Kang ${ }^{1}$ and \\ Chang Soo $\mathrm{Han}^{2 *}$ \\ ${ }^{1}$ Department of Mechatronic Engineering, Hanyang University, Seoul, Korea \\ ${ }^{2}$ Department of Mechanical Engineering, Hanyang University, Seoul, Korea \\ * Corresponding author (cshan@hanyang.ac.kr)
}

\begin{abstract}
Purpose Glass is widely used as finishing material for a good appearance of a building inside and outside. Moreover, the trend is to make this construction material and component larger and heavier. To safely and easily install this heavy duty and fragile glass, we propose an easy handling robot (EHR) system for glass installation work. Method We used an intuitive installation method; robot analysis using robotics; robot design; dynamic analysis using DAFUL. Results \& Discussion The EHR-system is divided into mobile, manipulator, and human robot cooperation (HRC) algorithm parts. We designed the mobile and manipulator, we then verified using DAFUL. We verified the HRC-algorithm by experiment. It is expected that the EHR-system will make this type of work easier and safer.
\end{abstract}

Keywords: robotics, intuitive installation method, virtual axis, robot design, robot analysis

\section{INTRODUCTION}

Installation work of large outer wall panel glasses such as curtain walls in construction sites is carried out at the border between the inside and outside of a building. This kind of work increases the labor load and stress of workers and the danger of such accidents as falling and crane overturning. Until now, such large glass finishing materials have been installed by workers. Except for the movement of glasses, all the work processes including assembly and installation have depended on workers ${ }^{1}$. This is because there is a limitation in completely automating glass installation works (access to the installation position and fitting) in the changeable environment of construction sites. Furthermore, as the high-rise buildings become larger, it is an inevitable trend that such construction materials become larger and heavier, and installations of these materials have naturally increased. Therefore, as a measure to guarantee the safety of construction workers and shorten construction period, semiautomation systems based on human-robot cooperation (HRC) techniques instead of full automation are being developed and applied ${ }^{2 \sim 4}$.

OKTOPUS by Materials Handling Corp. in Australia, Mobile Ergonomic Handler by Arlington Equipment Corp. in the U.S., Geko \& Glass Robot Hire by GGR Corp. in the U.K., and KS Robot 280 by K.Schulten $\mathrm{GmbH} \&$ Co.KG. In Germany are representative glazing robot systems based on human-robot cooperation technology $y^{5 \sim 8}$. Similarly, $\mathrm{Yu}$ et al. in Korea developed a curtain wall robot with a 3DOF manipulator attached to the end-effector of a miniexcavator ${ }^{9}$. For these robots, the operator manipulates the joints of the robots. If unskilled operator uses this robot manipulation method, however, it takes long to work and has a high likelihood of accidents. To address this problem, Lee et al. developed a skylight glass installation robot with 6DOF F/T sensors so that operator can directly operate the robot from the robot end-effector ${ }^{10}$. Furthermore, Lee applied force control to promote the safety of workers and protect glasses when operator works from the robot end-effector ${ }^{11}$. Force control transmits the repulsive power of materials against the environment to the operator so that the operator can safely operate the robot for such tasks as assembly. However, additional problems occurred in actual application such as high sensor cost, calibration of the weight of heavy objects, and the separation of force and torque. Thus, Gil et al. proposed a robot installation method in which the operator defines the rotation axis of the panel glass (virtual axis) and the motion of glasses is determined by the force applied to the rotation axis and the force applied from a point of action on the panel glass ${ }^{12}$. This paper describes the design process of the Easy Handling Robot (EHR) system using this method.

The design process of EHR is as follows. First, the most appropriate model for EHR system is benchmarked through an analysis of the HRC algorithm which is the glass installation method proposed by Gil et al. and the construction work environment. Then, the permissible torque and speed of each joint is determined through kinematic and static analyses based on the rough appearance and motion of the model. Lastly, the decelerator, motor, and link structure of EHR are designed 
through the defined torque and speed. Figure 1 below illustrates the design process of EHR.

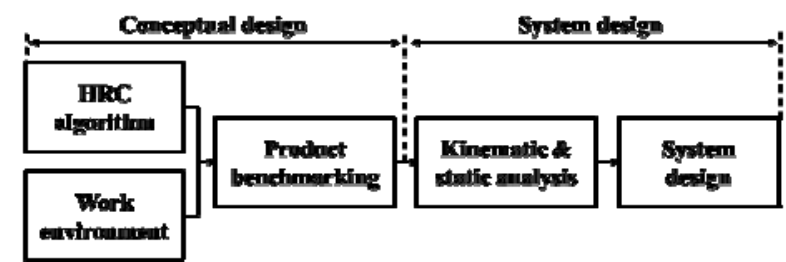

Fig. 1 Design process of EHR

\section{CONCEPTUAL DESIGN}

Work environment definition

The work environment for installation outer wall glass is shown in Figure 2. The panel glasses are transported to the installing position by passing through narrow passages and doors, rotating in a small space, and avoiding unexpected disturbances. Furthermore, the HRC algorithm proposed by Gil et al. is used.

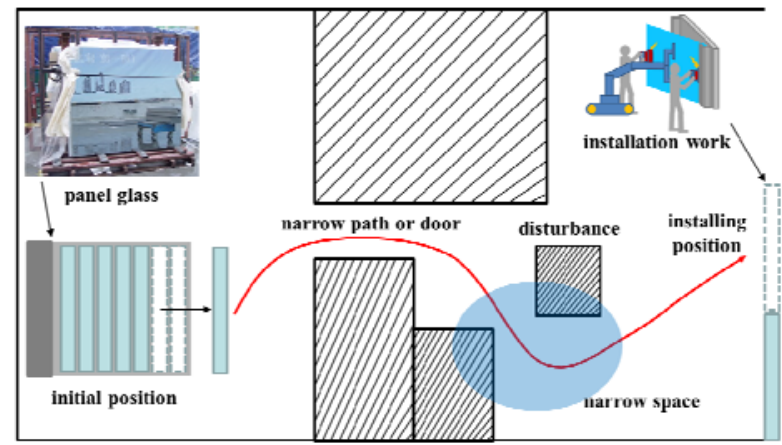

Fig. 2 Glass installation work

HRC algorithm

The HRC algorithm proposed by Gil et al. is described below. In general, the fundamental study of the HRC algorithm is the motion of a rigid body in space. To make it identical to the actual glass installation method by construction workers, the instantaneous axis of rotation and the point of action of the glass are defined. The robot outputs 6DOF motion from the end-effector that is mapped to the 3DOF linear motion which is inputted from these two axes.

Figure 3 shows the position of the instantaneous axis of rotation and demanded motion for worker to install panel glasses. As shown in Figure 3, the instantaneous axis of rotation changes three times in total until the glass is installed. For demanded motions, 3DOF linear motion (a), 3DOF rotational motion (b), and 1DOF rotational motion(c) are required.

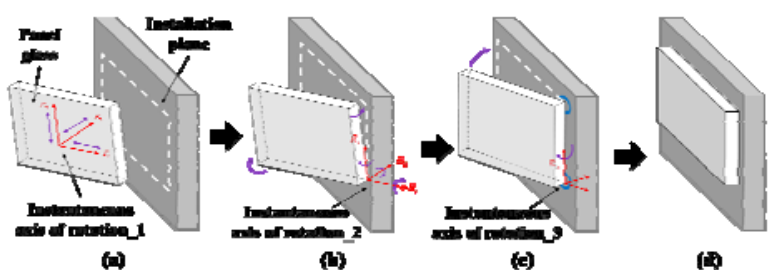

Fig. 3 The position of IR and required motion Product benchmarking

For the DOF and link structure of manipulator, one model of the existing commercial glazing robots was benchmarked considering the motions demanded for installing glasses to the outer wall. In this study, the Geko Pv+ model of GGR Corp. in the U.K. and the KS Robot 280 model of K.Schulten $\mathrm{GmbH}$ \& Co.KG. In Germany were compared, and the appropriate model for the EHR system was selected by the following process.

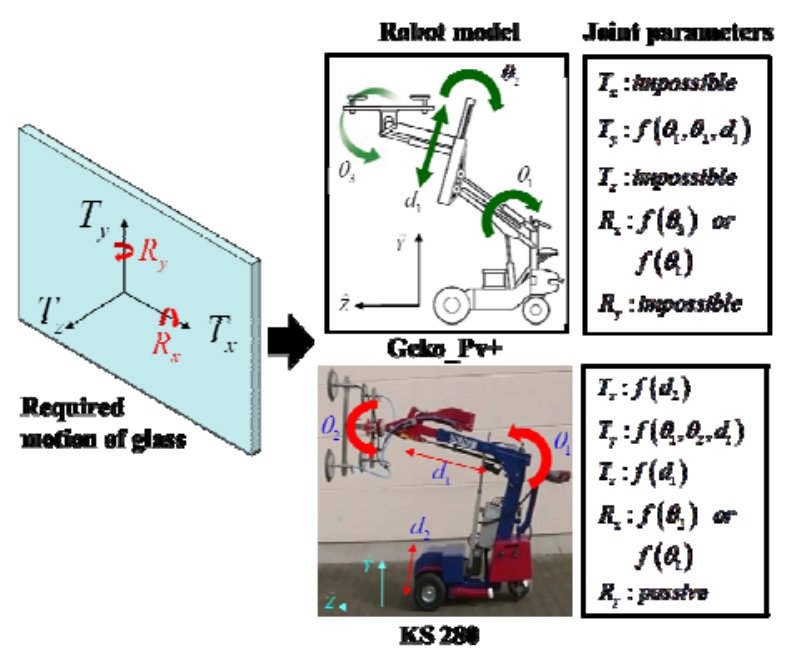

Fig. 4 Comparison of two models

The motion at the time when the panel glass is installed at the installing position is defined as follows. Under the assumption that the worker is working on a flat ground, we need $T_{x}, T_{y}$, and $T_{z}$ for 3-axis linear motion, and $R_{x}$ and $R_{y}$ for rotational motion. Figure 4 shows the demanded motion of the panel glass, the robot model, and the demanded joint parameters of the two robot models to implement the demanded motion. Here, "passive" means driving by worker with no motor. From Figure 4 , we can see that KS 280 is more appropriate for implementing the demanded motion of the panel glass than Geko Pv+. Therefore, the manipulator of the EHR system benchmarked the KS 280 model.

Conceptual design

Based on the work environment, HRC algorithm, and the benchmarking model, the requirements for the EHR system can be summarized as follows: 
1. The EHR system must be able to pass through a narrow door or passage during glass transportation.

2. It must be able to rotate in a narrow space.

3. It must be possible to install the panel glass on the outer wall.

4. The manipulator of the EHR system must have 5 DOF.

5. It must include the HRC algorithm.

6. It must benchmark the KS 280 model.

Figure 5 below shows the mobile part of the EHR system to satisfy the above conditions.

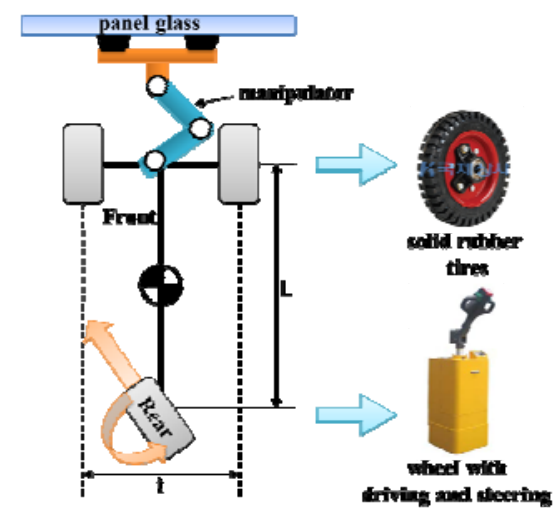

Fig. 5 Conceptual design of mobile part

The mobile part has three wheels (two fronts and one rear) to enable rotation in a narrow space. The steering wheel is behind the mobile part. The driving wheel is also located at the back to reduce the weight of the mobile part and to facilitate control. To allow the mobile part to pass through narrow passage or door, $\mathrm{t}$ is set to $600 \mathrm{~mm}$. Furthermore, to overcome nails and sharp scraps, the front wheels are made of solid rubber.

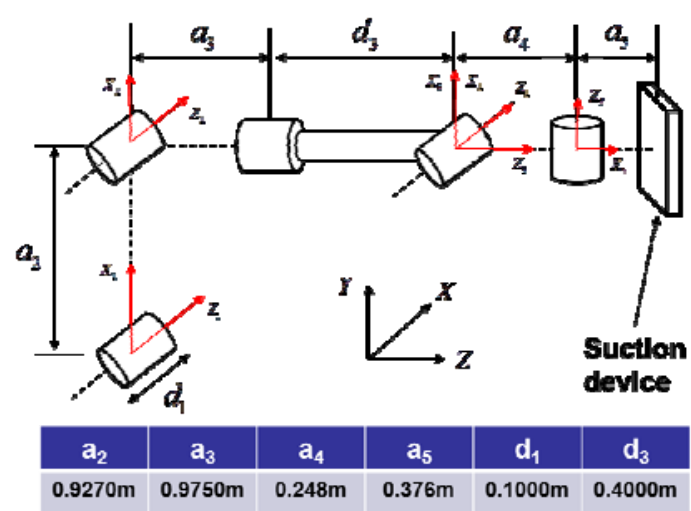

Fig. 6 Conceptual design of manipulator part

The manipulator part of the EHR system has 5 DOF (PRPRR type) to implement the HRC algorithm as shown in Figure 6. The length of each link of the manipulator is defined by benchmarking KS 280 . Considering that this is a prototype and for work safety, the payload of the manipulator was set to 100 $\mathrm{kg}$ and the speed at the end-effector was set to $0.03 \mathrm{~m} / \mathrm{s}$.

\section{DESIGN OF ROBOT SYSTEM}

This section describes the detailed design of the motor in line with the payload of the manipulator part and the speed of the end-effector.

\section{Design of manipulator part}

Axes 1 and 3 of the EHR system's manipulator are prismatic and axes 2, 4, and 5 are rotational. First, the axis that receives the largest torque among the rotational axes is 2 which is farthest from the panel glass. Thus, axis 2 was designed as a four bar linkage mechanism using a linear actuator as shown in Figure 7.

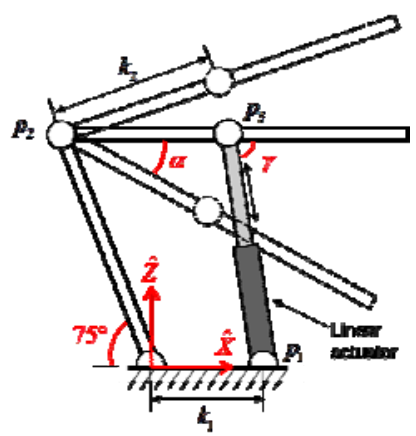

Fig. 7 Mechanism of 2nd axis

In the mechanism in Figure 7, the attachment position of the linear actuator affects the size of the load added to the actuator and the up and down speed of the robot end-effector. Thus, in this study, to minimize the load of the actuator and satisfy the demanded speed of the robot end-effector, the attachment position of the linear actuator was determined using the following optimization conditions.

First, $\mathrm{k}_{1}$ and $\mathrm{k}_{2}$ were defined roughly from the KS 280 model. Furthermore, $\alpha$ was defined by considering the average height of workers at the robot endeffector. The linear actuator must be as vertical to the ground as possible within the defined work range and the ratio of minimum to maximum lengths must not exceed 1.6. From these conditions, the optimization conditions are as follows:

$$
\begin{array}{ll}
\text { Find } & : k_{1}, k_{2} \\
\text { to minimize } & : F_{\max } \\
\text { subject to } & : \gamma<180^{\circ}, 1 \leq \frac{l_{\max }}{l_{\min }} \leq 1.6 \\
& 0.2 m \leq k_{1} \leq 0.45 \mathrm{~m} \\
& 0.4 m \leq k_{2} \leq 0.875 \mathrm{~m} \\
\text { condition } & :-38^{\circ} \leq \alpha \leq 11^{\circ} \\
& 8 \mathrm{~mm} / \mathrm{s} \leq v \leq 35 \mathrm{~mm} / \mathrm{s}
\end{array}
$$


From the above conditions, the following equations can be derived:

$$
\begin{aligned}
& l=\sqrt{\left(k_{1}+0.26125-k_{2} \cos (\alpha)\right)^{2}+\left(0.927+k_{2} \sin (\alpha)\right)^{2}} \\
& \gamma=A \tan 2\left(p_{1}(3)-p_{3}(3), p_{1}(1)-p_{3}(1)\right)-\alpha \\
& F=\frac{g \cos (\alpha)\left(M \times l_{c}+P \times 2.033\right)}{k \times|\sin (\gamma)|} \\
& v_{l}=\frac{\left|p_{2}-p_{3}\right| \mid \times \cos \times 1000}{|\sin (\gamma)|}
\end{aligned}
$$

where,

$$
\begin{aligned}
& p_{1}=\left[\begin{array}{l}
p_{1}(1) \\
p_{1}(2) \\
p_{1}(3)
\end{array}\right]=\left[\begin{array}{l}
k_{1} \\
0 \\
0
\end{array}\right], p_{2}=\left[\begin{array}{l}
p_{2}(1) \\
p_{2}(2) \\
p_{2}(3)
\end{array}\right]=\left[\begin{array}{c}
0.26125 \\
0 \\
0.927
\end{array}\right] \\
& p_{3}=\left[\begin{array}{l}
p_{3}(1) \\
p_{3}(2) \\
p_{3}(3)
\end{array}\right]=\left[\begin{array}{c}
k_{2} \cos (\alpha)-0.26125 \\
0 \\
0.927+k_{2} \sin (\alpha)
\end{array}\right] \\
& k=\left|p_{1}-p_{3}\right| \\
& l: \text { length of linear actuator } \\
& F: \text { exerted force on linear actuator } \\
& y: \text { velocity of linear actuator } \\
& l,: \text { mass center of upper link } \\
& P: \text { exerted force on robot end-effector } \\
& M: \text { mass of upper link } \\
& \omega: \text { angular velocity }
\end{aligned}
$$

\begin{tabular}{|c|c|c|c|c|c|c|}
\hline$k_{1}$ & $k_{2}$ & 7 & I & $\frac{l_{\max }}{l_{\min }}$ & $F_{n x}$ & $v_{\operatorname{mox}}$ \\
\hline $0.2 m$ & $0.4797 \mathrm{~m}$ & $\begin{array}{l}-01.14 \div Y \\
\leq-44.48\end{array}$ & $\begin{array}{l}0.6372 m \leq i \\
51.0188 m\end{array}$ & 1.6868 & $\begin{array}{c}7884,0 N \\
\text { (sta:lo) }\end{array}$ & $\begin{array}{c}20.2892 \\
\text { mints }\end{array}$ \\
\hline
\end{tabular}

Using the PQRSM (progressive quadratic response surface method) of the integrated optimization software PIAnO of FRAMAX Corp., we can derive the design parameters from the above equations as shown in Table 1.

Table 1 Design parameter of $2^{\text {nd }}$ joint mechanism

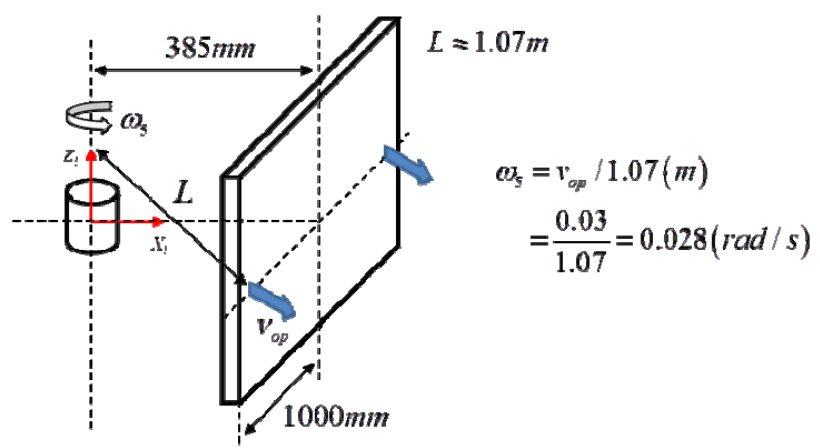

Fig. 8 Angular velocity of $5^{\text {th }}$ axis

In the case of axis 5, the angular speed is proportionate to the linear speed inputted by operator. If the glass size is $2(\mathrm{~m}) \times 1.8(\mathrm{~m}) \times 10(\mathrm{t})$, the linear speed inputted by operator and the angular speed at the axis 5 are as shown in Figure 8.
Furthermore, if the payload of EHR is $100 \mathrm{~kg}$, the suction device is about $60 \mathrm{~kg}$, and the distance from the axis 5 to the center of gravity of the glass is 240 $\mathrm{mm}$, the torque required for 5 axis is about $377 \mathrm{Nm}$. In addition, to maintain the current position with the manipulator stopped, the reduction gears of axis 5 are selected in the order of figure 9 to transmit power.

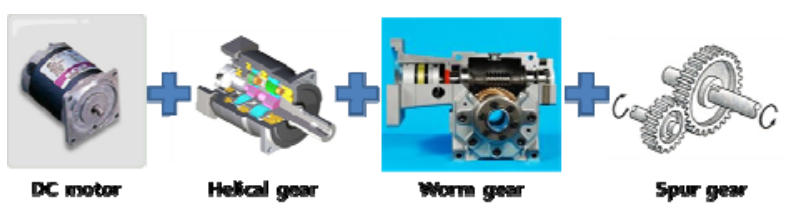

Fig. 9 Motor and reduction of $5^{\text {th }}$ axis

In the above figure, the worm gear model has the permissible torque of $196 \mathrm{Nm}$ at the maximum output of $9 \mathrm{rpm}$. The reduction ratio of the spur gear is set to $2: 1$ by comparing the output torque of the worm gear with the maximum permissible torque. Furthermore, considering the efficiency of the worm gear, the reduction ratio of the helical gear and the torque of the DC motor are defined as follows:

$\left[\begin{array}{l}\tau_{\text {out_hel }}=\tau_{\text {out_DC }} \cdot R_{\text {hel }} \cdot \lambda_{\text {hel }} \\ v_{\text {out_hel }}=v_{\text {out_DC }} / R_{\text {hel }}\end{array} \rightarrow\left[\begin{array}{l}4.4688(\mathrm{Nm})=0.1862 \cdot 30 \cdot 0.8 \\ 100(\mathrm{rpm})=3000 / 30\end{array}\right.\right.$
$\left[\begin{array}{l}\tau_{\text {out_worm }}=\tau_{\text {out_hel }} \cdot R_{\text {worm }} \cdot \lambda_{\text {worm }} \\ v_{\text {out_worm }}=v_{\text {out_hel }} / R_{\text {worm }}\end{array} \rightarrow\left[\begin{array}{l}196(\mathrm{Nm})=4.4688 \cdot 100 \cdot 0.5 \\ 1(\mathrm{rpm})=100 / 100\end{array}\right.\right.$
$\left[\begin{array}{l}\tau_{\text {out_spur }}=\tau_{\text {out_worm }} \cdot R_{\text {spur }} \cdot \lambda_{\text {spur }} \\ v_{\text {out_spur }}=v_{\text {out_worm }} / R_{\text {spur }}\end{array} \rightarrow\left[\begin{array}{l}384(\mathrm{Nm})=196 \cdot 2 \cdot 0.98 \\ 0.5(\mathrm{rpm})=1 / 2 \rightarrow 0.0524(\mathrm{rad} / \mathrm{s})\end{array}\right.\right.$

For axis 4, a chain sprocket and a linear actuator were used to prevent interference with 3 and 5 links and to allow driving with the minimum torque.

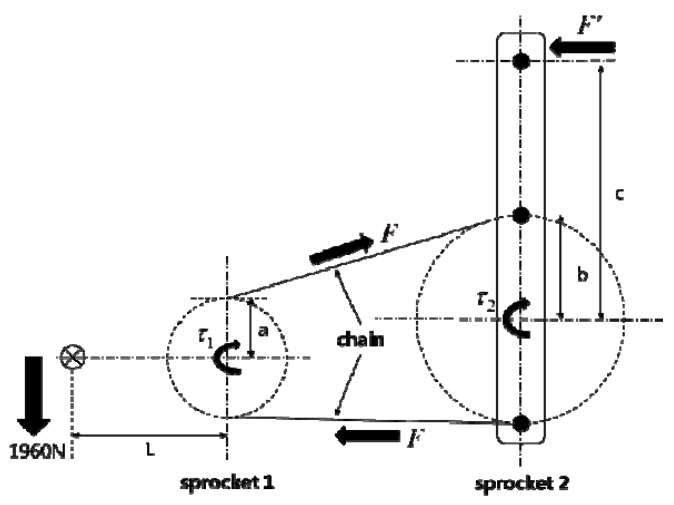

Fig. 10 Mechanism of $4^{\text {th }}$ axis

In Figure 10 above, if $L$ is $500 \mathrm{~mm}$ and $200 \mathrm{~kg}$ is applied, the torque on sprocket 1 is $882 \mathrm{Nm}$. Considering the number of teeth of sprocket 1 and the minimum fracture load of the chain, the RS50 double-row chain was selected. The force applied to the chain is $23105 \mathrm{~N}$ and the number of teeth of sprocket 1 is 15 . Furthermore, the number of teeth of 
sprocket 2 is set to 23 considering the demanded speed, motor speed, and torque.

Axis 1 supports the load of manipulator and glass and implements linear motion. Appropriate parts must be selected considering the moment and friction due to the load. Thus, an LM guide was used to support the load of manipulator and glass and linear motion was enabled using ball screws. The positions of ball screws and motor must not interfere with the mobile platform. Figure 11 below shows the prismatic joint of $1^{\text {st }}$ axis.

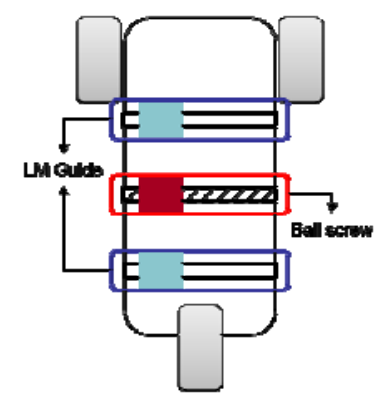

Fig. $111^{\text {st }}$ prismatic joint

The permissible torque and speed of axis 1 are defined as follows. If $\mathrm{N}$ is the number of runs, $\mathrm{L}$ is the running distance, and $v_{\mathrm{L}}$ is the maximum speed,

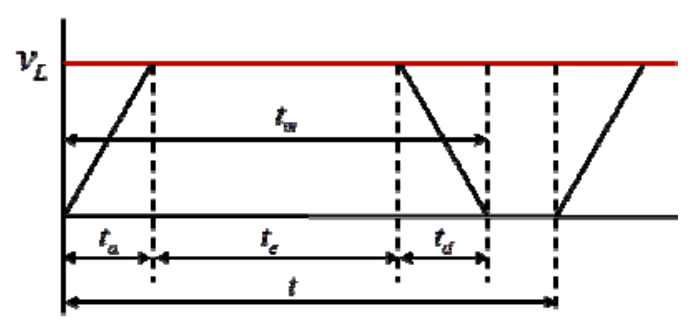

$t=\frac{60}{N} \quad t_{a}=t_{m}-\frac{60 \times L}{v_{L}}$

as $v_{\mathrm{L}}$ is $0.03 \mathrm{~m} / \mathrm{s}(1.8 \mathrm{~m} / \mathrm{min})$,

$t_{a}=t_{m}-\frac{60 \times L}{v_{L}}=5-\frac{60 \times 0.1}{1.8}=1.7 \mathrm{sec}$

$t_{c}=5-1.7 \times 2=1.6 \mathrm{sec}$

If the external diameter of ball screw is $20 \mathrm{~mm}$, and the lead is $5 \mathrm{~mm}$, the thrust torque and speed of the ball screw are as follows:

$T=\frac{F_{a} \cdot \text { lead }}{2 \pi \cdot \eta} \cdot \mu=\frac{300 \cdot 9.8 \cdot 0.005}{2 \cdot \pi \cdot 0.9}=0.0078(\mathrm{Nm})$

lead $=\frac{v_{\max }}{N_{\max }} \rightarrow N_{\max }=\frac{1.8(\mathrm{rpm} / \mathrm{min})}{0.005(\text { lead })}=360 \mathrm{rpm}$

Here, $F_{a}$ is the vertical load, $\eta$ is efficiency, and $\mu$ is the coefficient of friction.

In the case of axis 3 , the force applied to the prismatic joint varies by the working range of axis 2 .
Assuming that there is no friction force and a load of about $200 \mathrm{~kg}$ is acting on the robot end-effector, the force applied to the prismatic joint according to the working range of axis 2 can be illustrated as Figure 12 below.

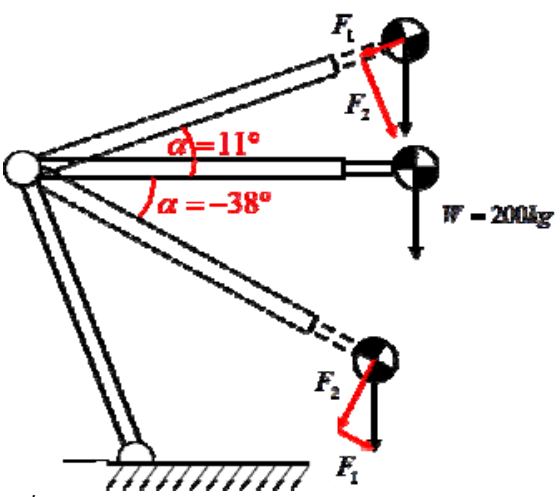

Fig. $123^{\text {rd }}$ prismatic joint

From Figure 12, the force $F_{1}$ on the 3 prismatic joint can be expressed as follows:

$\left[\begin{array}{l}\alpha=11^{\circ} \rightarrow F_{1}=374 N \\ \alpha=-38^{\circ} \rightarrow F_{1}=1207 N\end{array}\right.$

Furthermore, the maximum speed was set to $0.03 \mathrm{~m} / \mathrm{s}$ if only 3 joint is driven.

\section{Design for assembly}

The EHR system including the motor, reduction gears, and link structures is shown in Figure 13 below.

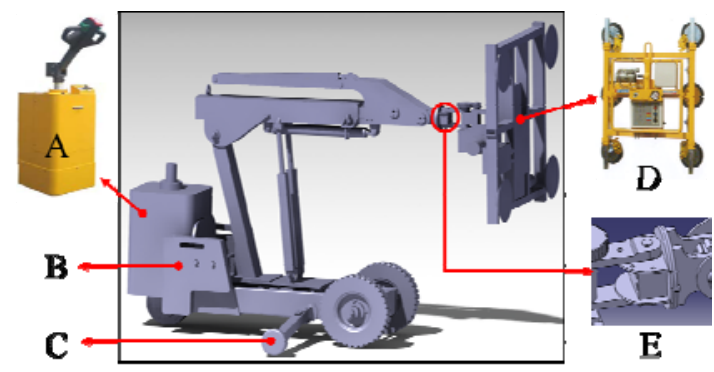

Fig. 13 EHR design for assembly

In Figure 13, part A (wheel with driving and steering) and $D$ (suction device) are existing commercial products. Part $\mathrm{B}$ is a weight balancer for balancing the center of gravity of the EHR system during work. Part $C$ is an outrigger to prevent the overturning in the transverse direction. Part $E$ is a passive joint to narrow the width of EHR system when passing through a narrow door with a glass attached.

\section{SIMULATION}

This section describes a simple simulation about overturning with the multi-objet simulation software DAFUL of Virtual Motion. If it is assumed that $100 \mathrm{~kg}$ is applied to the robot end-effector, the overturning likelihood of the EHR system can be determined 
through the moment of the front wheels in static situation.

Figure 14 below shows the locations of vertical load on the robot and the acting position.

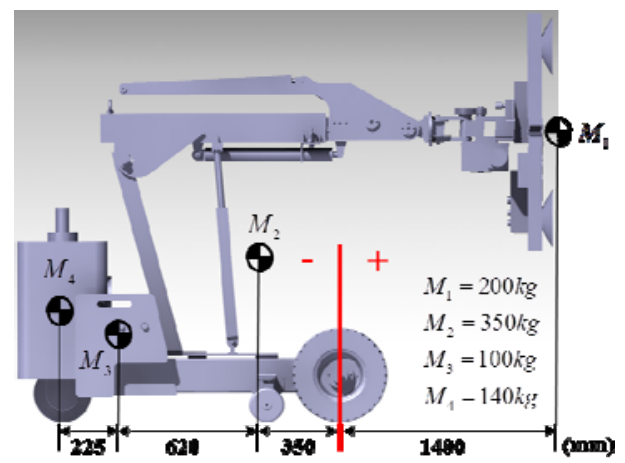

Fig. 14 Vertical load and position of vertical load

In Figure $14, M_{1}$ is the center of gravity when assuming that the weights of glass, suction device, and axis 5 are acting on the robot end-effector. $M_{2}$ is the center of gravity of axes 1 4 and the mobile platform. $M_{3}$ is the center of gravity of the weight balancer. Furthermore, $M_{4}$ is the center of gravity of part $A$ in Figure 13. The moment acting on the front wheels is as follows:

$$
\begin{aligned}
M & =1400 \times 200-(350 \times 390+970 \times 100+1195 \times 140) \\
& =-120800(\mathrm{~kg} \cdot \mathrm{mm})
\end{aligned}
$$

The above result shows that the moment in static situation is negative (-) and stable against overturning. Next, Figure 15 below shows the simulation of a dynamic situation using DAFUL.

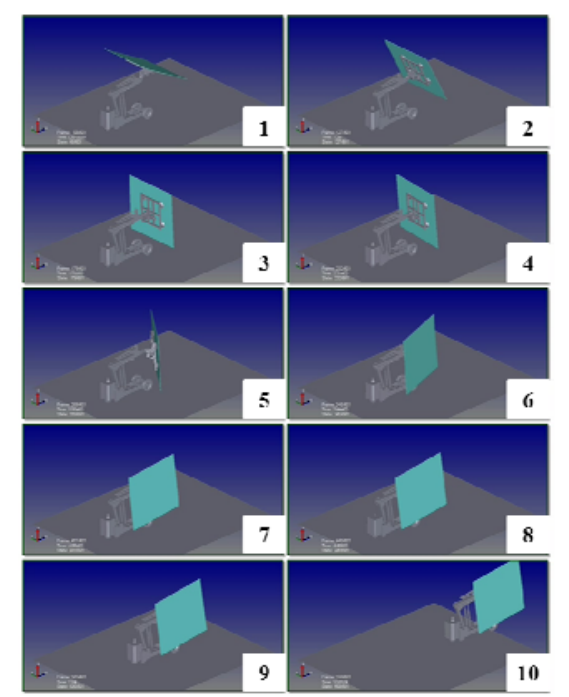

Fig. 15 Simulation for dynamic motion

In Figure 15, sections 1-3 show $90^{\circ}$ rotation of axis 4 , sections 4-7 show $90^{\circ}$ rotation of axis 5 , and sections 8-10 show the motion of mobile movement. The floor was assumed to be dry asphalt.
The simulations of static and dynamic situations described above show that the EHR system is stable against overturning.

\section{CONCLUSION}

Installation of outer wall glasses in construction sites is a type of work with a high risk of accident which causes high labor load and stress to workers. Thus, many glazing robots are being commercialized. This paper described the design of an EHR system that has been improved the problems of such glazing robots and can be used intuitively even by unskilled workers.

The design process of the EHR system was as follows. First, the restraints of the robot system were defined from the construction work environment and the HRC algorithm proposed by Gil et al. Next, based on these restraints, the most appropriate model for the EHR system among the existing glazing robots was benchmarked. Then, the permissible torque and speed of each joint were determined through kinematic and static analyses based on the rough appearance and motion of the model. The permissible torques and speeds of these joints became the restraints for designing the motor, reduction gears, and link mechanisms. The mobile driving parts and suctions parts were replaced with commercial products. Finally, the entire system was verified using the multi-object simulation software DAFUL. It was first verified against overturning in static situation and then in dynamic situation. The simulation results showed that the proposed system is stable against overturning during mobile movement. More studies are required in the future to verify the performance of each joint through simulations, the actual application of the robot system, and the proposed HRC algorithm.

\section{ACKNOWLEDGEMENT}

This research was supported by Basic Science Research Program through the NRF funded by the Ministry of Education, Science and Technology(2010-0025255), the Industrial Strategic technology development program (10040180) funded by the Ministry of Knowledge Economy and BMRC supported KICTEP under the MLTM.

\section{References}

1. Balaguer, C., Mohamed A., "Trends in Robotics and Automation in Construction", ISBN 978-953-761913-8, InTech, 2008.

2. Fukuda T., Fujisawa Y., Muro E., Hoshino H., Miyazaki T., Uehara K., Ohtsubo K. and Mikami T., "A New Robotic Manipulator in Construction based on Man-Robot Cooperation Work", Proc. Of the 8th ISARC, pp. 239 - 2445

3. Fukuda T., Fujisawa Y., Kosuge K., Arai F., Muro E., Hoshino H., Miyazaki K., Ohtsubo K., Uehara K., 
"Manipulator for Man-Robot Cooperation", International Conference on Industrial Electronics, Control and Instrumentation, Vol. 2, pp. 996 1001.

4. Fukuda T., Fujisawa Y., Arai F., "Study on ManRobot Cooperation Work-type of Manipulator", 1st Report, Mechanism and Control of Man-Robot Cooperation Manipulator, Trans. Of the JSME, pp. $160-168$

5. OKTOPUS, "Materials handling",

www.materialshandling.com.au/, Australia, Materials Handling Co.

6. Mobile Ergonomic Handler, "Home of the original Mobil Ergonomic Handler", www.gotoartech.com, U. S.A, Arlington Equipment Co.

7. Geko \& Glass Robot Hire, "GGR Glass", www.ggrglass.co.uk, England, GGR Co.

8. KS 280, http://www.ksschulten.com/de/index.html, Germany, K. Schulten Gmbh \& Co.KG

9. Seung-Nam Yu, Seung-Yel Lee, Chang-Soo Han, Kye-Young Lee, Sang-Heon Lee, "Development of the curtain wall installation robot: Performance and efficiency tests at a construction site", Autonomous Robots, pp.281-291, 2007

10. Seungyeol Lee, Myeongsu Gil, Kyeyoung Lee, Sangheon Lee, Changsoo Han, "Design of a Ceiling Glass Installation Robot", International Symposium on Automation and Robotics in Construction (ISARC 2007), pp.247-252, 2007

11. Seung-Yeol Lee, Kye Young Lee, Sang Heon Lee, Jin Woo Kim, Chang Soo Han, "Human-robot cooperation control for installing heavy construction materials", Autonomous Robots, Vol. 22, pp. 305319, 2007

12. Myeong-Su Gil, Min-Sung Kang, Seung-Hoon Lee and Chang-Soo Han, "Proposal of installation method of heavy duty glass using intuitive manipulation device", ISARC 2011, pp.76-82, 2011 